\title{
Jean-Christian Vinel, The Employee. A Political History
}

Philadelphia, University of Pennsylvania Press, coll. « Politics and

Culture in Modern America », 2013

\section{Claude Didry}

\section{OpenEdition}

\section{Journals}

Édition électronique

URL : http://journals.openedition.org/travailemploi/8753

DOI : $10.4000 /$ travailemploi.8753

ISSN : 1775-416X

Éditeur

DARES - Ministère du Travail

\section{Édition imprimée}

Date de publication : 1 juillet 2018

Pagination : 165-173

ISSN : 0224-4365

\section{Référence électronique}

Claude Didry, «Jean-Christian Vinel, The Employee. A Political History », Travail et Emploi [En ligne],

155-156 | Juillet-décembre 2018, mis en ligne le 11 juillet 2019, consulté le 25 septembre 2020. URL : http://journals.openedition.org/travailemploi/8753 ; DOI : https://doi.org/10.4000/travailemploi.8753 


\title{
Notes DE LECTURE
}

\section{The Employee. A Political History}

\author{
Jean-Christian Vinel
}

Philadelphia, University of Pennsylvania Press, coll. « Politics and Culture in Modern America », 2013, 293 p.

\section{Claude Didry*}

La contestation de leur statut d'entrepreneur (contractor) par les chauffeurs de San Francisco affiliés à Uber, en 2014, a fait apparaitre que le statut de salarié ou, en suivant le droit américain, d'employee était un objet de revendication aux États-Unis, tant dans le cadre de mobilisations collectives que dans celui de procédures judiciaires (depuis la commission du travail jusqu' au tribunal fédéral). À la différence du contractor, qui est dégagé de tout lien avec son commanditaire une fois sa prestation accomplie, l'employee est titulaire d'un contrat de travail (employment contract). Certes, celui-ci est fréquemment considéré comme permettant une liberté absolue de rupture unilatérale de contrat, ce qui tranche avec les garanties entourant en France le contrat de travail à durée indéterminée. Mais, en dépit de sa sulfureuse réputation de «flexibilité », l'employment contract suppose quand même la prise en charge par l'employeur (employer) des frais engagés par l'employee dans l'accomplissement de son activité professionnelle. De plus, sa rupture ouvre aux salariés, du moins en Californie, l'accès au chômage indemnisé. Bref, cette affaire montre qu'au-delà des clichés entourant le salariat américain, il reste à découvrir un droit du travail que le partage entre législation fédérale, législations propres à chaque État et jurisprudences tend à rendre bien plus difficile à saisir que notre Code du travail servi par un systématisme propice à la clarté.

Cette revendication de la condition d'employee par les chauffeurs liés à des plateformes invite à la lecture de l'ouvrage de Jean-Christian Vinel sur l'histoire de cette catégorie dans le contexte du droit américain. Ce professeur d'histoire américaine à l'université Paris-Diderot est en effet l'auteur d'un ouvrage reconnu dans les milieux juridiques outre-Atlantique, tout autant pour son retour sur la genèse de cette catégorie à partir de la forme juridique que constitue l'employment contract, que pour sa description de l'accès au droit syndical qui s'y attache depuis la loi Wagner de $1935^{1}$.

\footnotetext{
* CNRS, Centre Maurice Halbwachs (CMH), ENS-EHESS.

1. La loi Wagner (du nom du sénateur qui l'a proposée) ou National Labor Relations Act est une loi adoptée en 1935 dans le cadre du New Deal. Elle prévoit une procédure de consultation des salariés devant conduire, pour une unité de négociation, à un référendum ouvrant la voie à une syndicalisation de l'ensemble des salariés appartenant à l'unité si la majorité en décide ainsi. L'objectif est d'aboutir à la négociation et à la signature d'accords collectifs. Cette loi
} 
Loin d'une histoire sociale tenant la lutte entre deux classes clairement établies pour le fondement d'un droit du travail visant à protéger les salariés contre les abus des employeurs, il s'agit ici de se demander ce qu'est un salarié, un employee, en faisant de cette catégorie même un enjeu de luttes sociales et politiques. Cela me conduit à proposer ici une lecture personnelle de ce livre, en y voyant un parallèle avec le cas d'une France marquée par le passage d'un régime de louage d'ouvrage où les ouvriers sont fréquemment considérés comme entrepreneurs, à un régime de salariat autour de la figure cruciale que constitue le contrat de travail ${ }^{2}$.

Sur cette base, l'ouvrage envisage les enjeux de la loyauté hiérarchique bloquant, pour les salariés investis de responsabilités, l'accès au droit de se syndiquer. La question a de fait donné lieu à une décision retentissante du National Labor Relations Board (NLRB) ${ }^{3}$ en 2006, qui a refusé ce droit aux infirmières organisant le service de leurs collègues. Poussant le critère de subordination à ses limites, la législation étatsunienne conduit ainsi à distinguer les employees « subordonnés » soumis à un devoir d'obéissance mais ayant le droit de se syndiquer et les managerial employees, c'est-àdire les employees « subordonnant » n'ayant donc pas vocation à se syndiquer. En ce sens, l'ouvrage interroge la catégorie d'employee, tant par un retour sur l'institution même de l'employment contract que par une analyse de la jurisprudence syndicale, qui trouve aujourd'hui un écho très important dans les actions des travailleurs de plateformes pour se voir requalifier en employees.

Cette actualité conduit à appréhender l'ouvrage de J.-C. Vinel de manière logique et chronologique : en envisageant d'abord l'émergence de la catégorie d'employee - la législation syndicale se fonde en effet sur cette catégorie pour définir ses «ayants droit »-avant de centrer l' analyse sur les managerial employees, ce que la contestation récente de la législation syndicale par les lobbies patronaux nous invite à faire.

\section{Retour sur la naissance et la signification de la catégorie d'employee (salarié)}

D'entrée de jeu, l'ouvrage de J.-C. Vinel surprend le lecteur habitué à une histoire sociale classique qui, dans le cas américain, envisage l'émergence du terme d'employee dans les années 1890 comme un prolongement euphémisé, à travers la notion d'employment at will, du servant tiré des lois britanniques sur les masters and servants. Ces lois ont considérablement renforcé la discipline industrielle en Angleterre entre les XVIII et XIX ${ }^{\mathrm{e}}$ siècles, en allant jusqu'à créer, en 1766, un délit de désertion pour un servant quittant son master sans le consentement de ce dernier.

prévoit également la création d'une autorité administrative, le National Labor Relations Board en charge de trancher les affaires concernant les actions d'entrave (unfair) des employeurs dans cette procédure de consultation.

2. Didry C. (2016), L'Institution du travail. Droit et salariat dans l'histoire, Paris, La Dispute.

3. Organe en charge d'arbitrer les litiges suscités par la mise en place d'unités de négociation syndiquées dans le cadre de la loi Wagner. 
De ce point de vue, elles ne sont pas sans évoquer l'ordre corporatif en France ${ }^{4}$. La notion d'employee suggère ainsi une forme modeste de libéralisation dans le contexte états-unien, à l'égard d'une base juridique britannique particulièrement contraignante. Cette libéralisation correspondait principalement au souci de rompre avec une législation évoquant l'esclavage, au lendemain de la guerre de Sécession, en aboutissant à la reconnaissance progressive de l'employment at will. Comme le souligne Simon Deakin $^{5}$ (p. 50) :

« Au début du $\mathrm{Xx}^{\mathrm{e}}$ siècle, presque tous les États avaient adopté la règle d'employment at will suivant laquelle le contrat de travail pouvait être rompu par l'une ou l'autre des parties sans préavis ni raison : le salarié n'avait donc aucune sécurité de l'emploi. »

Selon J.-C. Vinel, cette réflexion est au cœur de débats classiques entre les juristes américains. Les uns, comme Horace Wood dans son Treatise on the Law of Master and Servant, mais sous-titré Covering the Relations, Duties and Liabilities of Employers and Employees ${ }^{6}$, publié en 1877, sont tentés de simplement substituer le couple employerlemployee au couple master/servant. D'autres, comme Richard McMurtrie dans un article de 1893, soulignent l'ambiguïté de ces nouvelles catégories d'employer et d'employee en insistant sur la différence avec la relation d'autorité liant le master au servant.

Mais J.-C. Vinel propose une nouvelle lecture de cette histoire en suivant le cheminement du mot français employé dans les arrêts de la Cour suprême, entré dans le Webster Dictionary en 1849 et utilisé tel quel dans les arrêts de la Cour avant de se transformer progressivement en employee. Ce terme prend alors une signification large, en intégrant par exemple dans le recensement de 1890 des directeurs d'établissement, des ingénieurs, des employés qualifiés (au sens de clerks), mais aussi des travailleurs non qualifiés, des ouvriers à la pièce, des journaliers (p. 20). C'est alors moins par référence à la relation d'autorité liant le servant au master que par référence à l'indépendance de l'entrepreneur, du contractor, que se définit l'employee et que, conséquemment, se révèle l'employer. Cela renvoie dans l'ouvrage à l'arrêt Vane de 1889, faisant suite à un procès initié en 1884 par Vane, un électricien posant des lignes pour une compagnie télégraphique, la Bankers' and Merchants' Telegraph Company. Vane se fonde sur la législation de l'Indiana, son lieu de travail, pour assigner la compagnie et lui demander le remboursement de 13771 \$ engagés pour l'achat de matériels et le paiement de salaires à d'autres ouvriers, en revendiquant pour cela la qualité d' « employé » ainsi que l'on dit encore à l'époque. Finalement, Vane n'obtient pas gain de cause devant la Cour suprême, même si certaines juridictions lui avaient

4. Cottereau A. (2002), « Droit et bon droit. Un droit des ouvriers instauré, puis évincé par le droit du travail (France, $\mathrm{XIX}^{\mathrm{e}}$ siècle) », Annales. Histoire, sciences sociales, vol. 57, $\mathrm{n}^{\circ}$ 6, pp. 1521-1557.

5. Deakin S. (2010), « La contrainte au travail : une comparaison des systèmes de common law et de droit civil, $\mathrm{XVIII}^{\mathrm{e}}-\mathrm{XX} \mathrm{e}^{\mathrm{e}}$ siècles ", in Stanziani A., Le Travail contraint en Asie et en Europe. XVII ${ }^{e}-\mathrm{XX}{ }^{e}$ siècles, Paris, Éditions de la Maison des sciences de l'homme, pp. 35-70.

6. Je propose la traduction suivante, qui n'est pas toutefois complètement satisfaisante : « Traité de droit des maitres et des domestiques. Au sujet des relations, devoirs et responsabilités des employeurs et employés ». 
précédemment donné raison. La Cour suprême revient même ainsi sur sa jurisprudence, et notamment un arrêt de 1874 où elle avait jugé que les ouvriers d'un sous-traitant opérant pour le compte d'une compagnie de distribution d'eau devaient être considérés comme «employees » de la compagnie donneuse d'ordres ${ }^{7}$.

Il est ainsi possible de lire le premier chapitre de l'ouvrage comme établissant de façon inédite une sorte de préalable dans une histoire sociale tenant jusque-là le salariat pour un résultat immédiat du capitalisme. Aux États-Unis comme en France, l'enjeu est le passage progressif d'une division considérée comme naturelle du travail dans le cadre d'une agriculture quasi autarcique à une division industrielle du travail à l'échelle du pays. Dans cette perspective, l'employment at will évoqué dans le deuxième chapitre suppose que soient identifiés des employees avant que ne soit invoquée une liberté contractuelle absolue garantie à tout citoyen américain par la Constitution des États-Unis et justifiant le rejet de toute limitation légale de cette liberté. Le succès du boulanger Lochner contre une législation de l'État de New York limitant la durée du travail des enfants, dans un arrêt de la Cour suprême de 1905, est emblématique de cette époque où rien n'arrête la Cour dans l'affirmation de cette liberté, pas même des considérations ayant trait à la protection des enfants. C'est face aux dérives auxquelles aboutit la rigueur logique de la Cour suprême que s'organise ensuite une pensée de l'harmonie sociale autour d'un économiste-sociologue dont l'influence va se révéler cruciale dans les législations du travail à venir : John R. Commons.

La poussée de la pensée caractérisant l' «ère progressiste ${ }^{8}$ », dans laquelle il faut compter également - outre Commons - le « réalisme légal » d'un Roscoe Pound ou du juge Holmes, s'accompagne de réalisations importantes dans le domaine de la législation. Au niveau des États, des gouverneurs, comme le gouverneur Hughes dans l'État de New-York pendant les années 1910, tentent de promouvoir une protection de l'enfance et un salaire minimum. Mais, même en 1935, au lendemain de l'adoption de la loi Wagner reconnaissant un droit à l' organisation syndicale des travailleurs, demeure toujours la question de savoir si les travailleurs prétendant à ce droit sont des employees. J.-C. Vinel évoque sur cette question la situation des newsboys (livreurs de journaux) présentés en 1944 par leur employeur (le groupe Hearst), dans un contentieux sur le droit syndical, comme des independent contractors (p. 61). Cela le conduit à noter le pragmatisme de la Cour suprême qui répond alors au groupe Hearst que la notion d'employee « prend la couleur de ce qui l'entoure dans la réglementation ${ }^{9}$ au sein de laquelle elle apparaît, et tire sa signification du contexte de cette réglementation ${ }^{10}$ » (p. 61). En d'autres termes, la catégorie d'employee se dessine « en situation », au vu

7. Cette conclusion n'intervenait certes que de manière incidente, dans une affaire résultant de dommages causés par ces ouvriers à des tiers.

8. Époque allant des années 1880 aux années 1920, en rupture avec le laisser-faire de la période antérieure, le Gilded Age ou «période dorée » qui commence par la fin de la guerre de Sécession.

9. C'est-à-dire la loi Wagner.

10. Traduction personnelle. 
d'éléments matériels tels que l'organisation du travail et la relation durable avec un employer qui permettent de réfuter la qualification initiale d'independent contractor.

La question ne se pose pas au moment de la loi Wagner pour les contremaîtres (foremen), dont nous allons voir qu'ils sont un groupe décisif dans le devenir de cette législation. Comme le rappelle l'auteur, le contremaître demeure jusqu'à la veille de la Première Guerre mondiale dans une situation proche de celle des contractors (entrepreneurs) que nous avons rencontrés précédemment. Dans la plupart des secteurs industriels, il bénéficie d'une ligne budgétaire sur laquelle il lui revient de financer les membres de son équipe ${ }^{11}$, leur formation, leur équipement pour réaliser une tâche dont il fixe le rythme. Mais les progrès de la rationalisation du travail constatée des années 1900 aux années 1920 - notamment sous la forme emblématique du taylorisme conduisent à la remise en cause de la quasi-entreprise confiée au contremaître, en centralisant le recrutement du personnel et les achats de matériel. La conséquence, pour les contremaîtres, est un affaiblissement considérable de leur pouvoir par leur transformation en un rouage de l'organisation, ayant à relayer les normes et les objectifs posés par les ingénieurs au niveau des ateliers.

\section{Des « employees » aux « managerial employees », relais du pouvoir des propriétaires}

La diffusion de la catégorie d'employee au cours des deux premières décennies $\mathrm{du} \mathrm{XX}^{\mathrm{e}}$ siècle conduit à poser la question d'une représentation de ces employees liés à un même employeur, c'est-à-dire la question d'une démocratisation de l'entreprise conçue par Commons comme un going concern ${ }^{12}$. L'enjeu était alors pour Commons de sortir des schémas mettant la lutte des classes au centre des dynamiques sociales, que ce soit dans un sens conservateur et élitaire ou marxiste et prolétarien, en arrivant à créer des mécanismes de conciliation sur la base des dispositifs juridiques existants. Dans cette perspective, le « réalisme légal » de Pound et de Holmes, mais aussi et surtout l'institutionnalisme de Commons tendent à ramener la mécanique contractuelle à une construction juridique. Le problème n'est plus celui de la victoire d'une classe sur une autre, mais celui de la conception de dispositifs d'un nouveau genre permettant de surmonter les limites du contractualisme classique ${ }^{13}$. Sur cette base, la réflexion de Commons met au jour la dimension historique de la condition salariale dans un article de $1909^{14}$ décrivant le passage d'un cordonnier itinérant et aidant les paysans à faire leurs chaussures à des ouvriers d'usine. Auparavant, et plus particulièrement dans une conférence de 1900, Commons pose les bases d'un modèle de « gouvernement

11. Qu'il est allé recruter dans la rue en règle générale.

12. C'est-à-dire - dans la terminologie spécifique de Commons - un « devenir » partagé par les travailleurs et les employeurs.

13. Coutu M., Kirat T. (2012), « John R. Commons, Max Weber et les ordres juridiques de l'économie : les prémisses d'une sociologie économique du droit », Revue française de socio-économie, ${ }^{\circ}$ 9, pp. 209-225.

14. Commons J. R. (1909), "American Shoemakers, 1648-1895: A Sketch of Industrial Evolution", The Quarterly Journal of Economics, vol. 24, $\mathrm{n}^{\circ}$ 1, pp. 39-84. 
constitutionnel » de l'entreprise, à la manière du Parlement britannique dans lequel des représentants élus voisinent avec des représentants non élus. Cette pensée réformatrice va animer, pendant la première moitié du $\mathrm{Xx}^{\mathrm{e}}$ siècle, la fameuse « école du Wisconsin » dont sortiront de nombreux acteurs du New Deal.

De ces années 1930, qui voient émerger la législation syndicale, aux années 1960 qui voient le syndicalisme progresser dans les catégories moins probables des engineers et plus généralement chez les « cols blancs », c'est finalement la question de l'organisation qui semble dominer dans le travail tel que l'analysent les différentes générations de sociologues évoquées par J.-C. Vinel. La grammaire de cette époque est sans doute à rechercher dans le célèbre ouvrage d'Adolph Berle et Gardiner Means, The Modern Corporation and Private Property, qui diagnostique en 1932 une séparation croissante entre l'entreprise et son management d'une part, et les actionnaires de l'autre. Le champ syndical semble alors s'élargir à l'ensemble des employees au sein duquel techniciens et ingénieurs connaissent une progression importante. Mais ces dynamiques industrielles soulignées par nombre de sociologues et économistes américains s'accompagnent d'un mouvement souterrain : une contre-offensive patronale qui s'engage à partir de la réaction au mouvement des contremaîtres dès les années 1940 et se prolonge aujourd' hui par le démantèlement progressif du champ syndical américain.

À la fin des années 1930, le mouvement des contremaîtres, qui revendiquent le droit de se syndiquer, va susciter en réaction une véritable restauration du droit de propriété dont la légitimité a, aux yeux des grands actionnaires du pays, été mise à mal par la législation syndicale. La mobilisation initiale des contremaîtres prolonge la vague créée par la loi Wagner de 1935 dans l'industrie américaine et plus spécifiquement dans l'automobile, sous l'impulsion du United Auto Workers (UAW), le syndicat ouvrier. Cette loi institue une procédure d'accréditation syndicale conduisant à la syndicalisation obligatoire de l'ensemble des salariés d'une unité de négociation - ce peut être une équipe, un atelier, un établissement ou une entreprise - si une majorité du personnel y vote en faveur du syndicat. Les syndicats peuvent déposer des recours devant le National Labor Relations Board ${ }^{15}$ (NLRB) établi par la loi, en cas de pratiques déloyales (unfair labor practices) de la part des employeurs, par exemple lorsqu'ils tentent de bloquer leur campagne référendaire.

De fait, la reconnaissance d'une régulation syndicale des salaires se traduit par la fin des prérogatives des contremaîtres à l'égard des autres salariés (notamment ouvriers), déjà sérieusement démantelées par la rationalisation de l'organisation du travail. Dans le secteur automobile, les contremaîtres éprouvent alors un sentiment de déclassement qui les rapproche des ouvriers et les pousse à s'engager dans l'action syndicale. Thomas Dwyer, un des leaders du mouvement, employé chez Packard depuis 1905, ou son collègue James R. Wilkin, dans la même entreprise depuis 1909, ont en effet connu les transformations résultant du taylorisme (p. 92). Mais la création

15. Cet organisme correspondrait en France à l'actuelle Direction des relations du travail (DRT) dans sa compétence en matière d'accords collectifs et de représentativité. 
d'un syndicat des contremaîtres affilié aux grandes confédérations ouvrières American Federation of Labor (AFL) et Congress of Industrial Organizations (CIO) ne va pas de soi, compte tenu des antagonismes fonctionnels entre contremaîtres et ouvriers. La Foremen's Association of America (FAA), créée en 1942, ne s'affirme ainsi que progressivement tant dans l'obtention d'un soutien de la part du syndicalisme ouvrier que face aux entreprises de l'automobile.

La situation semble dans un premier temps aboutir à un succès pour les contremaîtres, avec une décision du NLRB leur accordant le bénéfice du droit syndical chez Packard en 1945, confirmée par un arrêt de la Cour suprême en 1947. Cette évolution rappelle la dynamique des conventions collectives intégrant dans la France du Front populaire, à partir de 1936, les techniciens, agents de maîtrise et ingénieurs (p. 113) et dessinant un ensemble large de salariés (employees) par le regroupement des ouvriers et des « salaried workers » (on aurait dit dans la France de l'époque les «mensuels », c'est-à-dire les techniciens, ingénieurs et employés). Les différences hiérarchiques sont rapportées à une grille de classification commune, et justifiées par une organisation générale du travail, introduisant un degré d'ouverture beaucoup plus large dans les évolutions de carrière. La dimension primordiale est ici celle de la dépendance économique, c'est-à-dire la dépendance à l'égard de la poursuite de l'emploi soumise au bon vouloir de l'employeur, plutôt que celle de la subordination reposant sur un pouvoir de directive lié davantage à la professionnalité des encadrants qu'au pouvoir tiré de la détention du capital.

Mais cette dynamique qui se poursuit en France - des classifications fixées par les conventions collectives du Front populaire aux catégories Parodi-Croizat à la Libération, et se retrouve dans la nomenclature de l'Institut national de la statistique et des études économiques (Insee) des catégories socioprofessionnelles ${ }^{16}$ - est remise en cause outre-Atlantique par la loi Taft-Hartley aménageant en 1947 le régime syndical instauré en 1935. Le principal argument justifiant cette loi, et que fourbissent à l'envi les représentants du lobby des grandes entreprises, est qu' « un homme ne peut pas servir deux maîtres ${ }^{17} \gg$. Dans cette perspective, le règne des propriétaires - soit d'un capital par actions, soit d'un patrimoine familial - comme masters, implique d'identifier des relais de cette autorité dans le personnel et les contremaîtres en représentent l'incarnation la plus emblématique. Il en résulte pour ces derniers une obligation de loyauté qui tient à leur proximité avec le management, vue comme incompatible avec le droit de se syndiquer. C'est sur cette base que la loi Taft-Hartley introduit pour l'employeur le droit d'agir contre des « pratiques anti-employeur » et plus particulièrement de demander que la possibilité de se syndiquer ne s'applique pas à des travailleurs relevant de la catégorie des managerial employees.

\footnotetext{
16. Amossé T. (2013), « La nomenclature socioprofessionnelle : une histoire revisitée », Annales. Histoire, sciences sociales, vol. $68, \mathrm{n}^{\circ} 4$, pp. 1039-1075.

17. «A man can't serve two masters » (p. 1).
} 
La loi Taft-Hartley marque un point d'inflexion dans la législation du travail, avec un retour des actionnaires annonçant cette affirmation de la corporate governance (gouvernement d'entreprise) que nous connaissons aujourd'hui. Les contremaîtres ont constitué un premier terrain d'affrontement pour délimiter la population des managerial employees. Ils ont ouvert la voie à de nombreuses autres catégories de salariés, les « cols blancs » (employés, techniciens, ingénieurs) liés à la montée des qualifications. Ainsi, en considérant les chargés d'affaires de Bell Aerospace, qui sont au cœur du monde des ingénieurs, comme des managerial employees, une décision du NLRB rendue en 1971 et confortée par la Cour suprême en 1974 leur refuse le droit de se syndiquer. Comme l'indique J.-C. Vinel, cela n'empêchera toutefois pas l'ouverture d'une négociation dans cette entreprise en 1976, le rapport de forces prévalant sur les injonctions judiciaires.

Il faut noter ici l'une des vertus de l'ouvrage, celle de présenter une histoire ouverte dans laquelle la conclusion n'est pas inéluctablement la victoire du conservatisme. Si la législation syndicale a profondément été altérée par la loi Taft-Hartley, celle-ci ne détermine pas totalement le devenir du syndicalisme, comme on le voit dans le cas Bell Aerospace. De plus, l'alternance des majorités présidentielles conduit à des évolutions sensibles dans la jurisprudence du NLRB et de la Cour suprême avec, par exemple, une ouverture du droit à la syndicalisation et à la négociation collective aux fonctionnaires par la législation des États sous les présidences Kennedy et Johnson puis, en sens inverse, un resserrement sous la présidence Nixon.

Si l'issue de ces débats reste incertaine, une série de décisions rendues par le NLRB en 2006 a marqué les esprits et suscité une forte émotion, en classant des infirmières dans le groupe des managerial employees au motif qu'elles organisent le travail dans certains services (cf. supra). Cette décision a en effet remis en cause une mobilisation de plusieurs décennies : en 1972, au terme d'une lutte politique intense, une première loi avait réintégré les infirmières dans le champ de la législation syndicale après qu'elles en eurent été exclues par la loi Taft-Hartley au nom de l'exercice d'un « pouvoir discrétionnaire » dans leurs activités ; il avait fallu alors argumenter pour n'y voir que l'exercice d'un « jugement professionnel », relevant de leur expertise paramédicale et non de leur position d'encadrement. Dans les années 2000, le NLRB dominé par des membres issus de l'administration Bush revient sur cette interprétation et assimile les infirmières à des managerial employees, en semant un doute profond sur la portée du droit syndical issu de la loi Wagner. L'inflexion conservatrice du NRLB le conduit à intégrer une part croissante des salariés dans la catégorie des managerial employees et ainsi à les exclure du droit syndical.

L'ouvrage de J.-C. Vinel présente le grand intérêt de montrer que le droit du travail n'est pas une ressource réservée aux salariés et à leurs représentants. Sa mobilisation, ainsi que les aménagements que celle-ci suggère à des fins d' " amélioration », se retrouve également dans les activités managériales, ce qui peut donner lieu à une managérialisation du droit entendue tout à la fois comme son appropriation par des 
experts proches des employeurs et la recherche d'une reconfiguration législative visant à rendre le droit plus adapté aux intérêts de ces derniers ${ }^{18}$.

Cette managérialisation se présente sous un jour profondément conservateur, en accompagnant la restauration d'un capitalisme de propriétaires, ramenant le travail salarié aux emplois requis par ces derniers. La situation états-unienne tend à redéfinir la logique de la subordination selon une certaine réminiscence des lois Masters and Servants qui émane de l'argumentation actuelle des grandes entreprises américaines, en suggérant un partage du personnel entre des salariés subordonnés et des salariés subordonnant. Ainsi, la catégorie américaine de managerial employee, en ramenant l'organisation à une ligne hiérarchique, tend à ébranler profondément l'unité des employees, au moment où le développement des plateformes tend à repousser de nombreux travailleurs susceptibles d'être considérés comme des employees « non manageriaux » vers la condition de contractors.

18. Edelman L. B. (2016), Working Law. Courts, Corporations and Symbolic Civil Rights, Chicago, University of Chicago Press. 